\title{
Incommensurate Phonon Anomaly and the Nature of Charge Density Waves in Cuprates
}

\author{
H. Miao, ${ }^{1, *}$ D. Ishikawa, ${ }^{2,3}$ R. Heid, ${ }^{4}$ M. Le Tacon, ${ }^{4}$ G. Fabbris, ${ }^{1}$ D. Meyers, ${ }^{1}$ \\ G. D. Gu, ${ }^{1}$ A. Q. R. Baron, ${ }^{2}$ and M. P. M. Dean ${ }^{1,+}$ \\ ${ }^{1}$ Condensed Matter Physics and Materials Science Department, Brookhaven National Laboratory, \\ Upton, New York 11973, USA \\ ${ }^{2}$ Materials Dynamics Laboratory, RIKEN SPring-8 Center, RIKEN, Sayo Hyogo 679-5148, Japan \\ ${ }^{3}$ Research and Utilization Division, SPring-8/JASRI, Sayo Hyogo 679-5148, Japan \\ ${ }^{4}$ Institut für Festkörperphysik, Karlsruher Institut für Technologie, \\ P.O. Box 3640, D-76021 Karlsruhe, Germany
}

(Received 4 May 2017; revised manuscript received 25 November 2017; published 18 January 2018)

\begin{abstract}
While charge density wave (CDW) instabilities are ubiquitous to superconducting cuprates, the different ordering wave vectors in various cuprate families have hampered a unified description of the CDW formation mechanism. Here, we investigate the temperature dependence of the low-energy phonons in the canonical CDW-ordered cuprate $\mathrm{La}_{1.875} \mathrm{Ba}_{0.125} \mathrm{CuO}_{4}$. We discover that the phonon softening wave vector associated with $\mathrm{CDW}$ correlations becomes temperature dependent in the high-temperature precursor phase and changes from a wave vector of 0.238 reciprocal lattice units (r.l.u.) below the ordering transition temperature to 0.3 r.l.u. at $300 \mathrm{~K}$. This high-temperature behavior shows that " 214 "-type cuprates can host CDW correlations at a similar wave vector to previously reported CDW correlations in non-214-type cuprates such as $\mathrm{YBa}_{2} \mathrm{Cu}_{3} \mathrm{O}_{6+\delta}$. This indicates that cuprate CDWs may arise from the same underlying instability despite their apparently different low-temperature ordering wave vectors.
\end{abstract}

DOI: 10.1103/PhysRevX.8.011008

Subject Areas: Condensed Matter Physics, Strongly Correlated Materials

\section{INTRODUCTION}

Strongly correlated materials, such as the hightemperature superconducting cuprates, exhibit remarkably rich phase diagrams, due in large part to strong coupling between their charge, spin, and lattice degrees of freedom. Measuring how phonons are modified in the presence of CDWs can therefore provide compelling insights about the detailed CDW properties [1-11]. Early work examining phonon dispersion in the cuprates tended to focus on $\mathrm{Cu}-\mathrm{O}$ bond-stretching and bond-buckling phonon modes and identified a strong broadening and softening with cooling around $(0.25,0)$ in reciprocal lattice units (r.l.u.) [1-5,7-9]. These phonon anomalies have been widely discussed in terms of fluctuating stripes or CDWs that were originally predicted theoretically soon after the discovery of high- $T_{c}$ superconductivity [12-15] and that are thought to freeze into an ordered state via coupling to octahedral tilts in the lattice [16]. More recently, cuprate CDWs have gained

\footnotetext{
hmiao@bnl.gov

'mdean@bnl.gov
}

Published by the American Physical Society under the terms of the Creative Commons Attribution 4.0 International license. Further distribution of this work must maintain attribution to the author(s) and the published article's title, journal citation, and DOI. renewed consideration because of the discovery of shortranged CDW correlations in essentially all $1 / 8$ doped cuprates [11,17-24], with a large phonon anomaly occurring on the transverse acoustic and optical phonon modes [11]. While the presence of these correlations indicates that they may be an intrinsic property of superconducting cuprates, there are notable differences in the phenomenology [25-27]. Static CDW order in 1/8 doped lanthanum-based " 214 "type cuprates emerges at low temperatures around $(0.25,0)$, with an incommensurability that appears locked to the spin density wave (SDW) correlations around $(0.375,0.5)$ (i.e., with an incommensurability of $1 / 8$ relative to the antiferromagnetic wave vector). For this reason, CDWs in 214-type materials are often called "stripes" in analogy to phases in insulating nickelates $[16,28]$. Stripe formation is thought to be driven primarily by frustrated spin interactions induced by doping a Mott insulator [12-15]. CDWs in non-214 cuprates appear at different wave vectors [e.g., $(0.3,0)$ for $\mathrm{YBa}_{2} \mathrm{Cu}_{3} \mathrm{O}_{6+\delta}$ (YBCO)]. This wave vector shows no clear relationship with the location of the low-energy spin excitations, which tend to be gapped. Non-214 cuprates also have smaller incommensurabilities at higher doping levels, whereas 214 systems tend to follow the Yamada relationship where the CDW wave vector increases with doping in concert with changes in the SDW incommensurability $[17,29]$. Such differences pose a significant challenge to a unified description of CDWs in all cuprates. 
Here, we report phonon-dispersion measurements of archetypal CDW-ordered $\mathrm{La}_{2-x} \mathrm{Ba}_{x} \mathrm{CuO}_{4}, x=0.125$, and $x=0.095[30,31]$, in which we focus on the low-energy phonon modes associated with $c$-axis displacements complementing previously studied in-plane high-energy optical phonons $[2-5,8,9]$. The comparatively high spectral intensity of the low-energy modes, alongside progress in inelastic x-ray scattering (IXS) instrumentation [32,33], allows for measurements with excellent wave-vector $\boldsymbol{Q}$ and energy $\omega$ resolution and an extensive temperature dependence. For $x=0.125$, we find that CDW ordering at $55 \mathrm{~K}$ induces a small, narrow phonon anomaly near $(0.24,0)$, whereas short-ranged CDW correlations are established at much higher temperatures, giving rise to a broad phonon anomaly with a temperature-dependent wave vector that evolves from 0.24 r.l.u. below $55 \mathrm{~K}$ to 0.30 r.l.u. at $300 \mathrm{~K}$. This result has significant consequences. First, it shows that both the 214 and non-214 cuprate can host CDW correlations around 0.3 r.l.u. Second, combined with previous results [31], our data imply that CDW and SDW are unlocked at high temperatures. This behavior is captured by Landau theory modeling of CDW order, where a temperature-dependent wave vector arises because the CDW and SDW correlations, in isolation, minimize their energy at different wave vectors at high temperatures [34]. Upon cooling, coupling between the CDW and SDW becomes important and locks the CDW and SDW incommensurabilities together at low temperatures [34]. In summary, our measurement of a temperature-dependent CDW-related phonon softening thus indicates that cuprate CDWs can arise from the same underlying instability despite their apparently different ordering wave vectors.

\section{TWO ANOMALIES IN THE PHONONS}

An IXS measurement of the dynamic structure factor, $S(\boldsymbol{Q}, \omega)$, of $\mathrm{La}_{1.875} \mathrm{Ba}_{0.125} \mathrm{CuO}_{4}$ along with the phonon-mode dispersion predicted using density functional perturbation theory (DFPT) (Appendixes A and B and Ref. [35]) are shown in Fig. 1(a). Representative IXS raw data are shown in Figs. 1(b)-1(g). The spectra are dominated by two modes, referred to here as M1 and M2, in good agreement with the DFPT calculations. From the calculations, we ascertain that these modes are primarily associated with $z$ - and $y$-direction motions of the $\mathrm{La}$ and $\mathrm{Cu}$ atoms. M1 has primarily transverse acoustic character, and M2 has mixed longitudinal acoustic and longitudinal optical character. These modes connect to the transverse and longitudinal branches of the $[0,0, L]$ direction, respectively (see Ref. [35]).

In order to examine the temperature dependence of the signal, we plot the imaginary part of the phonon dynamic susceptibility $\chi^{\prime \prime}(\boldsymbol{Q}, \omega)$ in Fig. 2, which is derived by subtracting the elastic line and correcting for the thermal Bose factor as

$$
\chi^{\prime \prime}(\boldsymbol{Q}, \omega)=S(\boldsymbol{Q}, \omega)\left(1-e^{-\omega / k_{B} T}\right) .
$$

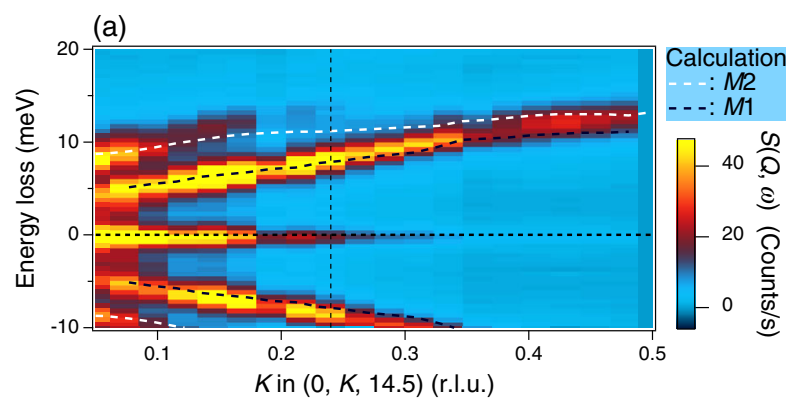

(b) $(0,0.09,14.5)$

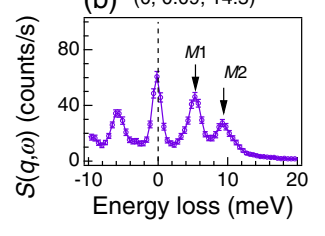

(e) $(0,0.23,14.5)$

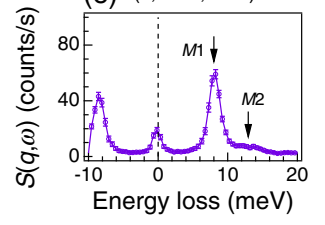

(c) $(0,0.15,14.5)$

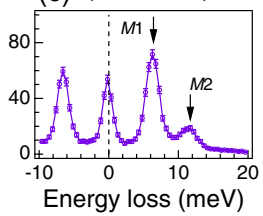

(f) $(0,0.31,14.5)$

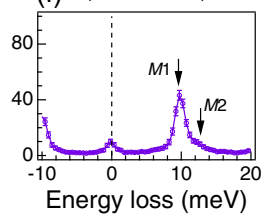

(d) $(0,0.2,14.5)$

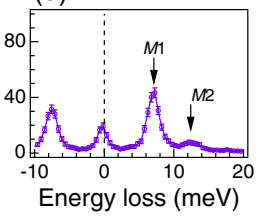

(g) $(0,0.34,14.5)$

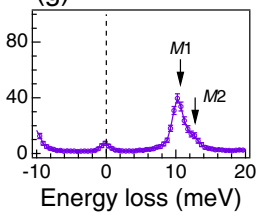

FIG. 1. Room-temperature phonon dynamic structure factor $S(\boldsymbol{Q}, \omega)$ of $\mathrm{La}_{1.875} \mathrm{Ba}_{0.125} \mathrm{CuO}_{4}$. (a) Color map around $(0, K, 14.5)$ for $K=0 \rightarrow 0.5$ r.l.u., showing two modes labeled $M 1$ and $M 2$ that match theoretical predictions shown as dashed lines. (b)-(g) Representative IXS spectra at $K=0.09,0.15,0.2$, $0.23,0.31$, and 0.34 r.l.u., respectively.

Panels (a)-(c) show spectra at $K=0.09,0.23$, and 0.31 r.l.u., respectively. A strong softening of M2 with decreasing temperature is seen at $\boldsymbol{Q}_{\mathrm{CDW}}$, which is reduced or absent at other $\boldsymbol{Q}$.

To extract the momentum- and temperature-dependent phonon properties, we fit all measured IXS spectra along $(0, K, 14.5)$. Phonon modes are represented by standard damped harmonic oscillator functions [36], where $A_{i}, \omega_{i}$, and $2 \gamma_{i}$ are the intensity, energy, and full width at half maximum (FWHM) of phonon peak $i$, which are weighted

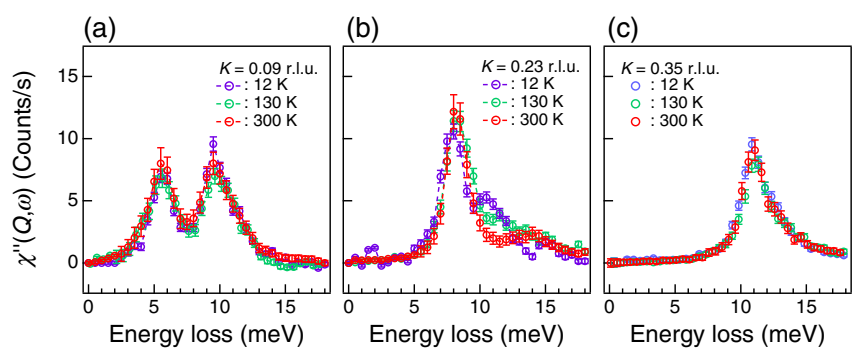

FIG. 2. Temperature dependence of the $\mathrm{La}_{1.875} \mathrm{Ba}_{0.125} \mathrm{CuO}_{4}$ phonon spectra. (a)-(c) Elastic-line-subtracted and Bose-factorcorrected IXS spectra at $K=0.09,0.23$, and 0.35 r.l.u., respectively. Purple, green, and red circles correspond to data at 12, 130, and $300 \mathrm{~K}$, respectively. Strong temperature dependence is seen at 0.23 r.l.u., whereas the $K=0.09$ and 0.35 r.l.u. show no obvious temperature dependence. 

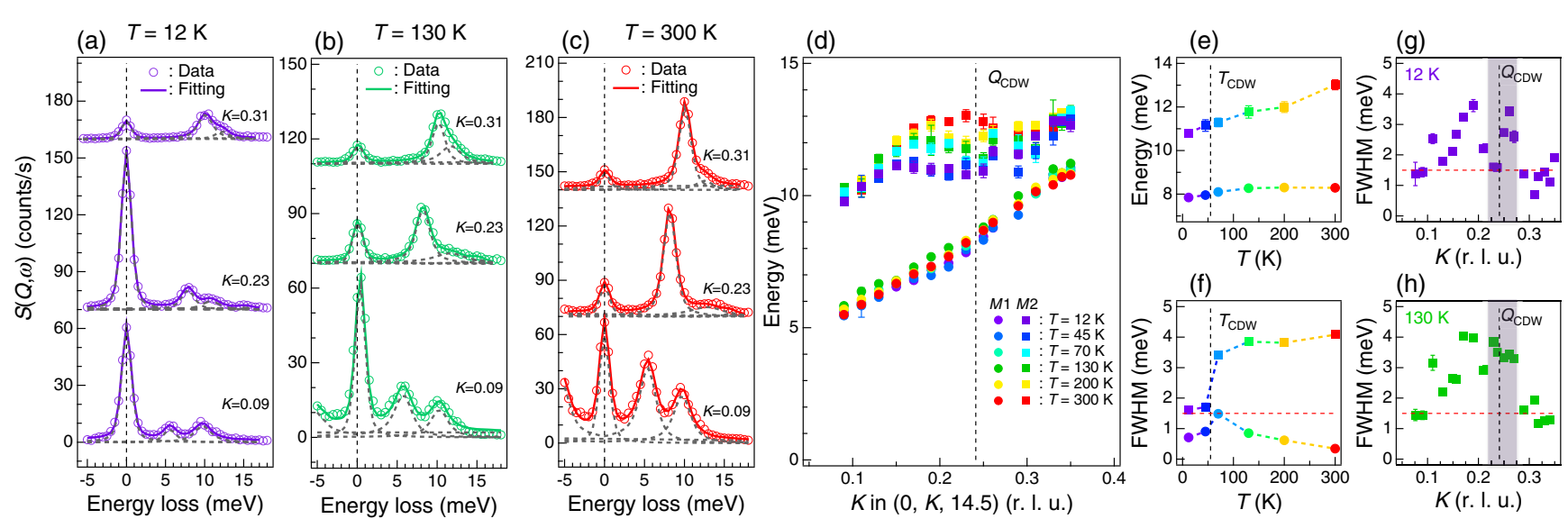

FIG. 3. Phonon line-shape analysis in $\mathrm{La}_{1.875} \mathrm{Ba}_{0.125} \mathrm{CuO}_{4}$. (a)-(c) Example fits of spectra at $K=0.09,0.23$, and 0.31 r.l.u. at temperatures of 12,130 , and $300 \mathrm{~K}$, respectively. The individual phonon and elastic components are shown as dashed gray lines. Spectra at $K=0.23$ and 0.31 r.l.u. are vertically shifted for clarity. (d)-(h) Evolution of fitting parameters. Modes $M 1$ and $M 2$ are shown as circles and squares, respectively, and different colors denote different temperatures. (d) The energy dispersion along $(0, K, 14.5)$ through $\boldsymbol{Q}_{\mathrm{CDW}}$ (vertical line). (e,f) Temperature-dependent peak energy and width, respectively, at $K=0.23$ r.l.u. $\left(\boldsymbol{Q}_{\mathrm{CDW}}\right)$. The vertical line marks $T_{\mathrm{CDW}}$. (g,h) The $\boldsymbol{Q}$-dependent peak widths at 12 and $130 \mathrm{~K}$, respectively. The shaded gray region at $\boldsymbol{Q}_{\mathrm{CDW}}$ shows the resolutionlimited width of the CDW Bragg peak at $12 \mathrm{~K}$. The horizontal red line denotes the experimental energy resolution of 1.5-meV FWHM. Note that width values do not include the experimental energy resolution, as this is separately accounted for by the convolution in Eq. (1) of Ref. [35].

by the Bose factor and convoluted with the $1.5-\mathrm{meV}$ energy resolution function [37]. An intensity-scaled resolution function is used to represent the elastic line (see Ref. [35]). We note that the enhanced elastic line seen at low temperatures around the CDW-ordering wave vector is energy-resolution limited, consistent with static CDW order [38]. Representative fitting results are shown in Figs. 3(a)-3(c), and the parameter evolution is plotted in Figs. 3(d)-3(h). M2 shows a strong softening over a broad $\boldsymbol{Q}$ range that is already visible at $200 \mathrm{~K}$ [Figs. 3(d) and 3(e)]. Based on the $\boldsymbol{Q}$ dependence of the effect, we assign it to phonons coupling to CDW correlations.

Figures 3(f)-3(h) show that M2 also tends to be strongly broadened by almost $4 \mathrm{meV}$ (FWHM) in a similar $\boldsymbol{Q}$ range to the softening, in contrast to $M 1$, which remains resolution limited at all temperatures. This broadening effect on $M 2$ is largely independent of temperatures from 300 to $70 \mathrm{~K}$, until a sharp reduction in the linewidth occurs upon cooling from 70 to $45 \mathrm{~K}$. This reduction in linewidth is localized to a small $\boldsymbol{Q}$ window around $\boldsymbol{Q}_{\mathrm{CDW}}$.

In view of the fact that $M 2$ couples more strongly to the CDW than $M 1$, we reexamined the displacements associated with these modes using DFPT (see Ref. [35]). Although at half-integer $L$, neither $M 1$ nor $M 2$ can be assigned to a purely longitudinal or transverse mode, we find that $M 2$ has a larger component of $c$-axis displacements compared to $M 1$. We therefore suggest that $c$-axis atomic displacements couple most strongly to the CDW. Because of the quasi-twodimensional electronic structure of the cuprates, $c$-axis displacements are expected to be much more weakly screened out, facilitating this stronger coupling. This conclusion is also consistent with studies that have associated cuprate CDWs with out-of-plane oxygen displacements $[16,39,40]$. Indeed, to reduce the strong interlayer Coulomb repulsion, the phase of the CDW in neighboring $\mathrm{CuO}_{2}$ layers is translated by $\pi$ to yield a CDW wave vector that is broadly peaked at half-integer $L$.

\section{RELATIONSHIP BETWEEN PHONONS AND CDW PROPERTIES}

How do we relate these anomalies in the lattice dynamics to the underlying CDW correlations in the cuprates? Upon cooling, $\mathrm{La}_{1.875} \mathrm{Ba}_{0.125} \mathrm{CuO}_{4}$ goes through a transition at $T_{\mathrm{CDW}}=55 \mathrm{~K}$, below which long-ranged (about $210 \AA$ ) static CDW order emerges [24,31,38,41-44]. The phonon narrowing effect outlined in Sec. II appears upon cooling below this transition [Fig. 3(f)], and its width in $Q$ is similar to that of the CDW Bragg peak [Fig. 3(g)]. We consequently associate the narrowing with the onset of the ordered CDW phase.

Phonon softening and broadening are, however, already apparent at temperatures well above $T_{\mathrm{CDW}}$. This has led previous $\mathrm{La}_{1.875} \mathrm{Ba}_{0.125} \mathrm{CuO}_{4}$ studies to speculate that the high-temperature phonon softening is associated with precursor $\mathrm{CDW}$ correlations, i.e., intrinsic fluctuating correlations that condense into the ordered state upon cooling $[4,6,9]$. The recent diffraction measurement of diffuse quasielastic scattering associated with precursor correlations in $\mathrm{La}_{1.875} \mathrm{Ba}_{0.125} \mathrm{CuO}_{4}$ [24] allows us to directly test the relationship between diffraction measurements of the CDW-order parameter and phonon softening. Figure 4 overlaps the phonon-softening and diffraction data seen in $\mathrm{La}_{1.875} \mathrm{Ba}_{0.125} \mathrm{CuO}_{4}$ and compares them to those observed 


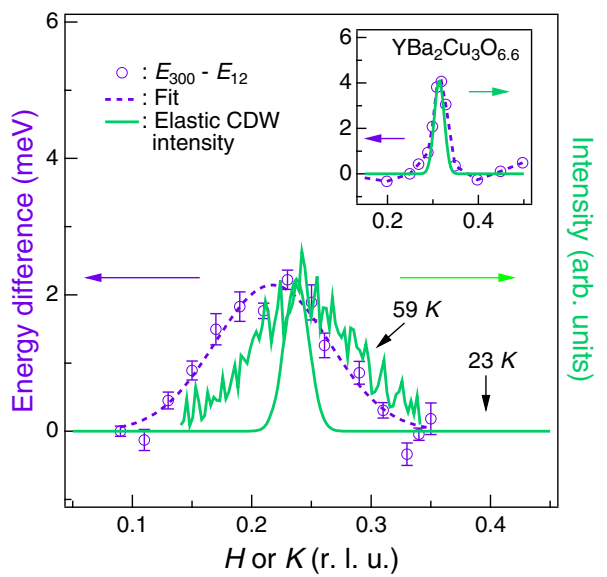

FIG. 4. Comparison between phonon softening and diffraction. Purple circles plot CDW-related phonon softening. The main panel plots the energy difference between 300 and $12 \mathrm{~K}$ for $\mathrm{La}_{1.875} \mathrm{Ba}_{0.125} \mathrm{CuO}_{4}$, whereas the inset shows the softening between 300 and $5 \mathrm{~K}$ for $\mathrm{YBa}_{2} \mathrm{Cu}_{3} \mathrm{O}_{6+\delta}$. Dashed purple lines are guides to the eye. These are compared to diffraction data in green. For $\mathrm{YBa}_{2} \mathrm{Cu}_{3} \mathrm{O}_{6+\delta}$ [11], the phonon softening mirrors the CDW Bragg peak. In $\mathrm{La}_{1.875} \mathrm{Ba}_{0.125} \mathrm{CuO}_{4}$, the phonon softening does not match the ordered CDW Bragg peak taken at $23 \mathrm{~K}$; rather, it matches precursor CDW measured at $59 \mathrm{~K}$ [24].

in cuprate $\mathrm{YBa}_{2} \mathrm{Cu}_{3} \mathrm{O}_{6+\delta}$ [11]. As seen in the inset, the $\mathrm{CDW}$-induced phonon softening closely matches the CDW Bragg peak in $\mathrm{YBa}_{2} \mathrm{Cu}_{3} \mathrm{O}_{6+\delta}$. In $\mathrm{La}_{1.875} \mathrm{Ba}_{0.125} \mathrm{CuO}_{4}$, however, the phonon softening is an order of magnitude broader in momentum space than the width of the CDW Bragg peak at $23 \mathrm{~K}$, suggesting it is not directly related to the CDW ordering. Instead, this broad phonon anomaly matches the width of the quasielastic precursor-CDW intensity observed by resonant inelastic x-ray scattering (RIXS) above $T_{\mathrm{CDW}}$ [24], explicitly confirming its association with the precursor CDW as distinct from the ordered phase. This is our first main observation.

Our results paint a different picture to that in conventional CDW systems [45-47]. These systems tend to exhibit phonons that soften to zero energy at the CDWordering transition, and such softening occurs in a relatively narrow temperature window around the transition. In some cases, these effects are reasonably well understood. For example, DFPT including strong electron-phonon coupling and phonon anharmonicity reproduces the phonon anomaly in $\mathrm{NbSe}_{2}$, including its broad momentum dependence, which has similar momentum width to that observed here [47]. One difference, however, is that in LBCO we observe only partial phonon softening, which occurs continuously from 300 to $12 \mathrm{~K}$. Combined with the fact that the phonon anomaly matches the width of the quasielastic precursor correlations, this result is more consistent with the idea that the anomaly reflects phonons coupling to the CDW correlations rather than electron-phonon coupling driving CDW formation [48]. In $\mathrm{La}_{1.875} \mathrm{Ba}_{0.125} \mathrm{CuO}_{4}$, the continuous softening, with only a very small phonon narrowing through the transition, may suggest a dominant role of the precursor CDW and that only a small fraction of the correlations are condensed below the transition temperature. Cuprates are known to host appreciable disorder [49,50], and, as theoretically modeled [51], disorder can suppress the formation of long-range ordered phases from short-range precursor correlations and may have an important role here.

\section{UNIVERSAL FEATURES OF CDWS IN CUPRATES}

A notable difference between $\mathrm{La}_{1.875} \mathrm{Ba}_{0.125} \mathrm{CuO}_{4}$ and other cuprates is the wave vector of the CDW correlations. Note that 214-type cuprates have wave vectors close to 0.25 r.l.u., whereas other $1 / 8$ doped cuprates tend to have higher wave vectors, such as 0.3 r.l.u. for $\mathrm{YBa}_{2} \mathrm{Cu}_{3} \mathrm{O}_{6+\delta}$ [25]. This motivated proposals that non-214-type cuprates should be understood using different paradigms than those in 214 systems $[17,19,25,52-54]$. In Fig. 5, we examine the phonon dispersion and the CDW-related phonon softening in more detail. The maximum phonon softening, which is close to the CDW-ordering wave vector at low temperatures, moves with increasing temperature, reaching around 0.3 r.l.u. at room temperature. Importantly, this unusual phonon softening is consistent with recent RIXS measurements of CDW-related quasielastic scattering in the same material [24], proving that the change of phonon softening is driven by the temperature-dependent CDW incommensurability. This is our second main observation. The strong precursor CDW correlation and its unusual temperature dependence can be compared to similar acoustic phonondispersion measurements in $\mathrm{YBa}_{2} \mathrm{Cu}_{3} \mathrm{O}_{6+\delta}$ [11]. Phonons in both $\mathrm{La}_{1.875} \mathrm{Ba}_{0.125} \mathrm{CuO}_{4}$ and $\mathrm{YBa}_{2} \mathrm{Cu}_{3} \mathrm{O}_{6+\delta}$ soften by a

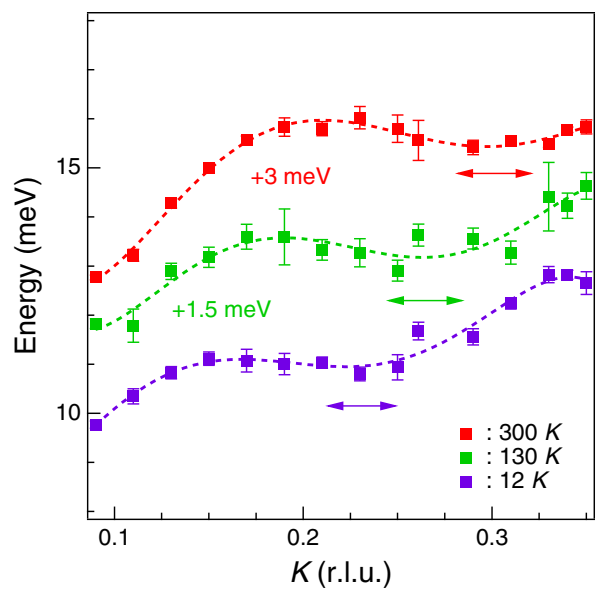

FIG. 5. Temperature-dependent phonon incommensurability in $\mathrm{La}_{1.875} \mathrm{Ba}_{0.125} \mathrm{CuO}_{4}$. Extracted phonon dispersions of $M 2$ at 12 , 130 , and $300 K$ along $(0 K 14.5)$ are shown as purple, green, and red squares. Dashed lines are guides to the eye, and arrows indicate the $\boldsymbol{Q}$ associated with the CDW-related phonon softening. Dispersions at 130 and $300 \mathrm{~K}$ are offset by 1.5 and $3 \mathrm{meV}$, respectively, for clarity. 
similar order of magnitude, $19 \%$ and $26 \%$, and both effects are already evident near 0.3 r.l.u. at the first temperature measured below the initial room-temperature measurement 200 and $150 \mathrm{~K}$, respectively. These comparable CDWassociated phonon anomalies are consistent with the similar CDW phase diagram in these two cuprate families, and this indicates, albeit circumstantially, that both CDWs may share a common origin.

\section{DISCUSSION OF STRONG-CORRELATION-INDUCED CDW}

In cuprates, the origin of the CDW is under debate between reciprocal space pictures such as Fermi-surface nesting and real space pictures with strong magnetic and Coulomb interactions. In the first class of scenarios, the most important property of the ground state is the wave vector, which nests regions with a high density of states on the Fermi surface, such as the antinodal Fermi surface $[17,54]$ or the end points of the Fermi arcs [19]. Here, the temperature dependence of the incommensurability (Sec. IV), as well as the broadness of the phonon softening in momentum space (Sec. II), does not match theoretical expectations based on the Fermi surface. These scenarios would predict that the CDW wave vector should either decrease at higher temperatures [55] or be temperature independent, neither of which is observed here. Instead, real space pictures are likely more important. These include those based on the competition between minimizing the number of broken magnetic bonds and kinetic energy and Coulomb repulsion between the doped holes [14,15, 56-59]. In such scenarios, the ordering wave vector arises from a balance between different ordering tendencies and is not a crucial defining parameter of the mechanism. These scenarios are more compatible with our observed variation of the CDW wave vector with temperature, which implies that the CDW wave vector is not solely defined by doping. Instead, it changes on a thermal energy scale of order $10 \mathrm{meV}$. It should be noted that a similar conclusion has been put forward based on STM studies of $\mathrm{Bi}_{2} \mathrm{Sr}_{2} \mathrm{CaCu}_{2} \mathrm{O}_{8+x}$, where the authors found that the local CDW wave vector is doping independent and that the change of the wave vector is due to "phase slips" in the CDW domain boundaries and hence not a fundamental property of the CDW [60].

\section{SPIN-CHARGE LOCKING IN La ${ }_{2-x} \mathrm{Ba}_{x} \mathrm{CuO}_{4}$}

Since underdoped cuprates have a large magnetic energy scale and a relatively small electronic density of states at the Fermi level, much of the early work on CDW and SDW ordered phases in the cuprates assumed a dominant spin degree of freedom [12-15,61]. Indeed, the low-temperature CDW/SDW ordered phases in 214 materials have incommensurabilities that follow the "Yamada" rule in which $\boldsymbol{Q}_{\mathrm{CDW}}=(2 \delta, 0)$ and $\boldsymbol{Q}_{\mathrm{SDW}}=(0.5-\delta, 0.5)$, with the incommensurability $\delta \approx x$. Here, we see that the CDW incommensurability increases with temperature. From previous inelastic neutron-scattering measurements of $\mathrm{La}_{1.875} \mathrm{Ba}_{0.125} \mathrm{CuO}_{4}$, we know that the SDW incommensurability decreases with temperature [31]. Thus, the spincharge locking is broken at high temperatures, showing that $\mathrm{La}_{2-x} \mathrm{Ba}_{x} \mathrm{CuO}_{4}$ can host a spin-charge unlocked phase very similar to non-214 cuprates, which also host CDWs with no related low-energy SDW correlations (the spin-excitation spectrum in $\mathrm{YBa}_{2} \mathrm{Cu}_{3} \mathrm{O}_{6+\delta}$ is, in fact, gapped). We further tested this by measuring $\mathrm{La}_{1.905} \mathrm{Ba}_{0.095} \mathrm{CuO}_{4}$ (plotted in the Supplemental Material Ref. [35]), finding that the maximum softening also occurs at larger wave vectors than the CDWordering wave vector of 0.21 r.l.u., again unlocked from the SDW wave vector [62]. All these results support the case for a universal CDW mechanism in the cuprates. Temperaturedependent incommensurabilities were actually predicted by Ginzberg-Landau-Wilson modeling of coupled CDW and SDW order parameters $[34,51,63]$. In such an approach, isolated CDW and SDW orders are assumed to minimize their energy at unrelated wave vectors, as we observe at high temperatures. Upon cooling, however, a cubic coupling term means that the CDW and SDW can save energy by locking their incommensurabilities in the ground state. We finally note that the low-temperature tetragonal (LTT) structural phase transition is also widely believed to have an important role for stabilizing CDW formation [16]. We suggest that the LTT distortion is likely to have a minimal role for locking the CDW and SDW here because (i) changes in the phonon wave vector are already apparent at temperatures well above the LTT transition [24,31] and (ii) the CDW in $\mathrm{La}_{2-x} \mathrm{Sr}_{x} \mathrm{CuO}_{4}$ also occurs around wave vectors of 0.24 r.l.u. despite the fact that this system does not exhibit a LTT transition $[21,64]$.

\section{CONCLUSIONS}

Our phonon-dispersion measurements of $\mathrm{La}_{1.875} \mathrm{Ba}_{0.125} \mathrm{CuO}_{4}$ reveal two CDW-related phonon anomalies: a phonon softening associated with precursor CDW fluctuations as well as a small phonon narrowing associated with CDW ordering. The phonon softening associated with precursor correlations occurs at a temperature-dependent wave vector changing from 0.238 to 0.3 r.l.u. as the system is heated from 12 to $300 \mathrm{~K}$. These results indicate that the precursor CDW reflects the intrinsic properties of the CDW correlations the are common to all underdoped cuprates, while the ordered phase reflects coupling between charge and spin correlations that locks the CDW and SDW together at low temperatures into a state that is particular to cuprates without a spin gap.

\section{ACKNOWLEDGMENTS}

M. P. M. D. and H. M. acknowledge Philip Allen, Chris Homes, José Lorenzana, Claudio Mazzoli, Jörg Schmalian, 
and John Tranquada for insightful discussions. IXS research by H.M. and M.P.M.D. is supported by the Center for Emergent Superconductivity, an Energy Frontier Research Center funded by the U.S. Department of Energy (DOE), Office of Basic Energy Sciences. Work at Brookhaven National Laboratory was supported by the U.S. DOE, Office of Science, Office of Basic Energy Sciences, under Contract No. DE-SC00112704. The synchrotron radiation experiments were performed at BL43LXU in SPring-8 with the approval of RIKEN (Proposals No. 20160097 and No. 20170076).

\section{APPENDIX A: SAMPLE GROWTH}

$\mathrm{La}_{2-x} \mathrm{Ba}_{x} \mathrm{CuO}_{4}$ single crystals were grown at Brookhaven National Laboratory using the floating zone method, and mmsized samples were prepared from the resulting rod by cutting and cleaving. The barium concentration of the samples was confirmed by checking the samples' superconducting transition via magnetization measurements. These are known to be around $2 \mathrm{~K}$ for $x=0.125$ and $31 \mathrm{~K}$ for $x=0.095[30,41]$. The crystallinity of the samples was checked both prior to and during the IXS measurement and found to be of excellent quality with crystal mosaics of about $0.02^{\circ}$. Numerous previous measurements on samples prepared in the same way further attest to the sample quality $[24,38,41-43,65]$. The wave vectors used in our manuscript are described using the high-temperature tetragonal $(I 4 / \mathrm{mmm})$ space group with $a=b=3.78 \AA$ and $c=13.28$ and $13.24 \AA$ for $x=0.125$ and $x=0.095$, respectively.

\section{APPENDIX B: EXPERIMENTAL SETUP}

High-precision phonon-dispersion measurements of $\mathrm{La}_{2-x} \mathrm{Ba}_{x} \mathrm{CuO}_{4}$ were made using the high-resolution IXS spectrometer installed at BL43LXU of SPring-8, Japan, which delivers world-leading $\mathrm{x}$-ray flux for an experiment of this type $[32,33]$. In order to maximize the effect on the phonons, we focus primarily on $x=0.125$ and measure the dispersion around $\boldsymbol{Q}_{\mathrm{CDW}}=(0,0.25,14.5)$, where a strong CDW Bragg peak develops below $T_{\mathrm{CDW}}=55 \mathrm{~K}$. A large $L$ geometry was chosen in order to enhance our sensitivity to $c$-axis displacements, which are only weakly screened because of the quasi-two-dimensional electronic structure of the cuprates. All data were taken with an incident energy around $21.75 \mathrm{keV}$ using the $(11,11,11)$ refection of silicon as a monochromator and analyzer. The instrumental resolution was found to be well described by a pseudo-Voight function

$R(\omega)=(1-\alpha) \frac{A}{\sqrt{2 \pi} \sigma} e^{-\left[\omega^{2} /\left(2 \sigma^{2}\right)\right]}+\alpha \frac{A}{\pi} \frac{\gamma}{\omega^{2}+\gamma^{2}}$,

where $\alpha$ is confined between 0 and 1 and controls the relative fraction of the Lorentzian and Gaussian functions; $\sigma$ and $\gamma$ characterize the width of the two functions, respectively. The overall width of this function was about 1.5-meV FWHM, which varies slightly depending on the analyzer.

The sample was mounted with the $\boldsymbol{H}$ and $\boldsymbol{L}$ reciprocal lattice vectors close to the horizontal scattering plane. In this configuration, a vertical column in the two-dimensional $(4 \times 6)$ analyzer array closely traced our desired $(0, K, 14.5)$ trajectory in reciprocal space $[7,33]$. Slits were used to define the momentum resolution of the measurement to be $(\delta \boldsymbol{H}, \delta \boldsymbol{K}, \delta \boldsymbol{L})=(0.02,0.02,0.2)$ r.l.u., where the relaxed value of $\delta \boldsymbol{L}$ was chosen in view of the known short correlation length of the CDW in this direction $[41,42]$. By examining spectra in the analyzers away from the main column of interest, we estimated the dispersion of $M 1$ and $M 2$ in the $L$ direction. From this estimate, we calculated that finite $\delta \boldsymbol{L}=0.2 \boldsymbol{Q}$ resolution contributes only about $0.25 \mathrm{meV}$ to the phonon linewidth, which is much smaller than the temperature-induced broadening effects discussed here. During the measurements, we interleaved multiple measurements such that neighboring points were separated by steps of 0.02 r.l.u. in $K$. Spectra were collected for about 1 hour at each momentum transfer. The efficiency of each analyzer was first approximately determined by measuring plexiglass and further refined $(<20 \%)$ by scaling the values to ensure that the signal evolves smoothly as a function of $\boldsymbol{Q}$.

[1] L. Pintschovius, N. Pyka, W. Reichardt, A. Yu Rumiantsev, N. L. Mitrofanov, A. S. Ivanov, G. Collin, and P. Bourges, Lattice Dynamical Studies of HTSC Materials, Physica C (Amsterdam) 185-189, 156 (1991).

[2] R. J. McQueeney, Y. Petrov, T. Egami, M. Yethiraj, G. Shirane, and Y. Endoh, Anomalous Dispersion of LO Phonons in $\mathrm{La}_{1.85} \mathrm{Sr}_{0.15} \mathrm{CuO}_{4}$ at Low Temperatures, Phys. Rev. Lett. 82, 628 (1999).

[3] H. Uchiyama, A. Q. R. Baron, S. Tsutsui, Y. Tanaka, W.-Z. Hu, A. Yamamoto, S. Tajima, and Y. Endoh, Softening of $\mathrm{Cu}-\mathrm{O}$ Bond Stretching Phonons in Tetragonal $\mathrm{HgBa}_{2} \mathrm{CuO}_{4+\delta}$, Phys. Rev. Lett. 92, 197005 (2004).

[4] D. Reznik, L. Pintschovius, M. Ito, S. Iikubo, M. Sato, H. Goka, M. Fujita, K. Yamada, G. D. Gu, and J. M. Tranquada, Electron-Phonon Coupling Reflecting Dynamic Charge Inhomogeneity in Copper Oxide Superconductors, Nature (London) 440, 1170 (2006); D. Reznik, T. Fukuda, D. Lamago, A. Q. R. Baron, S. Tsutsui, M. Fujita, and K. Yamada, Q-Dependence of the Giant Bond-Stretching Phonon Anomaly in the Stripe Compound $\mathrm{La}_{1.48} \mathrm{Nd}_{0.4} \mathrm{Sr}_{0.12} \mathrm{CuO}_{4}$ Measured by IXS, J. Phys. Chem. Solids 69, 3103 (2008).

[5] J. Graf, M. d'Astuto, C. Jozwiak, D. R. Garcia, N. L. Saini, M. Krisch, K. Ikeuchi, A. Q. R. Baron, H. Eisaki, and A. Lanzara, Bond Stretching Phonon Softening and Kinks in the Angle-Resolved Photoemission Spectra of Optimally Doped $\mathrm{Bi}_{2} \mathrm{Sr}_{1.6} \mathrm{La}_{0.4} \mathrm{Cu}_{2} \mathrm{O}_{6+\delta}$ Superconductors, Phys. Rev. Lett. 100, 227002 (2008). 
[6] M. d'Astuto, G. Dhalenne, J. Graf, M. Hoesch, P. Giura, M. Krisch, P. Berthet, A. Lanzara, and A. Shukla, Sharp Optical-Phonon Softening Near Optimal Doping in $\mathrm{La}_{2-x} \mathrm{Ba}_{x} \mathrm{CuO}_{4+\delta}$ Observed via Inelastic X-Ray Scattering, Phys. Rev. B 78, 140511 (2008).

[7] A. Q. R. Baron, J. P. Sutter, S. Tsutsui, H. Uchiyama, T. Masui, S. Tajima, R. Heid, and K.-P. Bohnen, First Study of the $b_{1 g}$ Buckling Phonon Mode in Optimally Doped, De-Twinned, $\mathrm{YBa}_{2} \mathrm{Cu}_{3} \mathrm{O}_{7-\delta}$ by Inelastic X-Ray Scattering, J. Phys. Chem. Solids 69, 3100 (2008).

[8] M. Raichle, D. Reznik, D. Lamago, R. Heid, Y. Li, M. Bakr, C. Ulrich, V. Hinkov, K. Hradil, C. T. Lin, and B. Keimer, Highly Anisotropic Anomaly in the Dispersion of the Copper-Oxygen Bond-Bending Phonon in Superconducting $\mathrm{YBa}_{2} \mathrm{Cu}_{3} \mathrm{O}_{7}$ from Inelastic Neutron Scattering, Phys. Rev. Lett. 107, 177004 (2011).

[9] D. Reznik, Phonon Anomalies and Dynamic Stripes, Physica C (Amsterdam) 481, 75 (2012).

[10] M. d'Astuto, I. Yamada, P. Giura, L. Paulatto, A. Gauzzi, M. Hoesch, M. Krisch, M. Azuma, and M. Takano, Phonon Anomalies and Lattice Dynamics in the Superconducting Oxychlorides $\mathrm{Ca}_{2-x} \mathrm{CuO}_{2} \mathrm{Cl}_{2}$, Phys. Rev. B 88, 014522 (2013).

[11] M. Le Tacon, A. Bosak, S. M. Souliou, G. Dellea, T. Loew, R. Heid, K-P. Bohnen, G. Ghiringhelli, M. Krisch, and B. Keimer, Inelastic X-Ray Scattering in $\mathrm{YBa}_{2} \mathrm{Cu}_{3} \mathrm{O}_{6.6}$ Reveals Giant Phonon Anomalies and Elastic Central Peak Due to Charge-Density-Wave Formation, Nat. Phys. 10, 52 (2014).

[12] J. Zaanen and O. Gunnarsson, Charged Magnetic Domain Lines and the Magnetism of High- $T_{c}$ Oxides, Phys. Rev. B 40, 7391 (1989).

[13] K. Machida, Magnetism in $\mathrm{La}_{2} \mathrm{CuO}_{4}$ Based Compounds, Physica C (Amsterdam) 158, 192 (1989).

[14] D. Poilblanc and T. M. Rice, Charged Solitons in the Hartree-Fock Approximation to the Large-U Hubbard Model, Phys. Rev. B 39, 9749 (1989).

[15] V. J. Emery, S. A. Kivelson, and H. Q. Lin, Phase Separation in the t-J Model, Phys. Rev. Lett. 64, 475 (1990).

[16] J. M. Tranquada, B. J. Sternlieb, J. D. Axe, Y. Nakamura, and S. Uchida, Evidence for Stripe Correlations of Spins and Holes in Copper Oxide Superconductors, Nature (London) 375, 561 (1995).

[17] G. Ghiringhelli, M. Le Tacon, M. Minola, S. BlancoCanosa, C. Mazzoli, N. B. Brookes, G. M. De Luca, A. Frano, D. G. Hawthorn, F. He, T. Loew, M. Moretti Sala, D. C. Peets, M. Salluzzo, E. Schierle, R. Sutarto, G. A. Sawatzky, E. Weschke, B. Keimer, L. Braicovich, M. Le Tacon, M. Minola et al., Long-Range Incommensurate Charge Fluctuations in $(\mathrm{Y}, \mathrm{Nd}) \mathrm{Ba}_{2} \mathrm{Cu}_{3} \mathrm{O}_{6+x}$, Science 337, 821 (2012).

[18] J. Chang, E. Blackburn, A. T. Holmes, N. B. Christensen, J. Larsen, J. Mesot, Ruixing Liang, D. A. Bonn, W. N. Hardy, A. Watenphul, M. V. Zimmermann, E. M. Forgan, and S. M. Hayden, Direct Observation of Competition Between Superconductivity and Charge Density Wave Order in $\mathrm{YBa}_{2} \mathrm{Cu}_{3} \mathrm{O}_{6.67}$, Nat. Phys. 8, 871 (2012).

[19] R. Comin, A. Frano, M. M. Yee, Y. Yoshida, H. Eisaki, E. Schierle, E. Weschke, R. Sutarto, F. He, A. Soumyanarayanan, Y. He, M. Le Tacon, I. S. Elfimov, J. E. Hoffman, G. A. Sawatzky, B. Keimer, and A. Damascelli,
Charge Order Driven by Fermi-Arc Instability in $\mathrm{Bi}_{2} \mathrm{Sr}_{2-x} \mathrm{La}_{x} \mathrm{CuO}_{6+\delta}$, Science 343, 390 (2014).

[20] E. H. da Silva Neto, P. Aynajian, A. Frano, R. Comin, E. Schierle, E. Weschke, A. Gyenis, J. Wen, J. Schneeloch, Z. Xu, S. Ono, G. D. Gu, M. Le Tacon, and A. Yazidani, Ubiquitous Interplay Between Charge Ordering and HighTemperature Superconductivity in Cuprates, Science 343, 393 (2014).

[21] V. Thampy, M. P. M. Dean, N. B. Christensen, L. Steinke, Z. Islam, M. Oda, M. Ido, N. Momono, S. B. Wilkins, and J. P. Hill, Rotated Stripe Order and Its Competition with Superconductivity in $\mathrm{La}_{1.88} \mathrm{Sr}_{0.12} \mathrm{CuO}_{4}$, Phys. Rev. B 90, 100510 (2014).

[22] M. Hashimoto, G. Ghiringhelli, W.-S. Lee, G. Dellea, A. Amorese, C. Mazzoli, K. Kummer, N. B. Brookes, B. Moritz, Y. Yoshida, H. Eisaki, Z. Hussain, T. P. Devereaux, Z.-X. Shen, and L. Braicovich, Direct Observation of Bulk Charge Modulations in Optimally Doped $\mathrm{Bi}_{1.5} \mathrm{~Pb}_{0.6} \mathrm{Sr}_{1.54} \mathrm{CaCu}_{2} \mathrm{O}_{8+\delta}$, Phys. Rev. B 89, 220511 (2014).

[23] W. Tabis, Y. Li, M. Le Tacon, L. Braicovich, A. Kreyssig, M. Minola, G. Dellea, E. Weschke, M. J. Veit, M. Ramazanoglu, A. I. Goldman, T. Schmitt, G. Ghiringhelli, N. Barišić, M. K. Chan, C. J. Dorow, G. Yu, X. Zhao, B. Keimer, and M. Greven, Charge Order and Its Connection with Fermi-Liquid Charge Transport in a Pristine High- $T_{c}$ Cuprate, Nat. Commun. 5, 5875 (2014).

[24] H. Miao, J. Lorenzana, G. Seibold, Y. Y. Peng, A. Amorese, F. Yakhou-Harris, K. Kummer, N. B. Brookes, R. M. Konik, V. Thampy, G. D. Gu, G. Ghiringhelli, L. Braicovich, and M.P.M. Dean, High-Temperature Charge Density Wave Correlations in $\mathrm{La}_{1.875} \mathrm{Ba}_{0.125} \mathrm{CuO}_{4}$ without SpinCharge Locking, Proc. Natl. Acad. Sci. U.S.A. 114, 12430 (2017).

[25] R. Comin and A. Damascelli, Resonant X-Ray Scattering Studies of Charge Order in Cuprates, Annu. Rev. Condens. Matter Phys. 7, 369 (2016).

[26] E. Fradkin, S. A. Kivelson, and J. M. Tranquada, Colloquium: Theory of Intertwined Orders in High Temperature Superconductors, Rev. Mod. Phys. 87, 457 (2015).

[27] B. Keimer, S. A. Kivelson, M. R. Norman, S. Uchida, and J. Zaanen, From Quantum Matter to High-Temperature Superconductivity in Copper Oxides, Nature (London) 518, 179 (2015).

[28] C. H. Chen, S-W. Cheong, and A.S. Cooper, Charge Modulations in $\mathrm{La}_{2-x} \mathrm{Sr}_{x} \mathrm{NiO}_{4+y}$ : Ordering of Polarons, Phys. Rev. Lett. 71, 2461 (1993).

[29] M. Fujita, Neutron Scattering Studies of Stripe Correlations in Curate Oxide Superconductors, Physica C (Amsterdam) 481, 23 (2012).

[30] A. R. Moodenbaugh, Y. Xu, M. Suenaga, T. J. Folkerts, and R.N. Shelton, Superconducting Properties of $\mathrm{La}_{2-x} \mathrm{Ba}_{x} \mathrm{CuO}_{4}$, Phys. Rev. B 38, 4596 (1988).

[31] M. Fujita, H. Goka, K. Yamada, J. M. Tranquada, and L. P. Regnault, Stripe Order, Depinning, and Fluctuations in $\mathrm{La}_{1.875} \mathrm{Ba}_{0.125} \mathrm{CuO}_{4}$ and $\mathrm{La}_{1.875} \mathrm{Ba}_{0.075} \mathrm{Sr}_{0.050} \mathrm{CuO}_{4}$, Phys. Rev. B 70, 104517 (2004).

[32] A. Q. R. Baron, RIKEN Quantum NanoDynamics Beamline (BLA3LXU): The Next Generation for Inelastic X-Ray Scattering, SPring 8 Information 15, 14 (2010). 
[33] A. Q. R. Baron, High-Resolution Inelastic X-Ray Scattering I: Context, Spectrometers, Samples, and Superconductors, in Synchrotron Light Sources and Free-Electron Lasers: Accelerator Physics, Instrumentation and Science Applications, edited by E. Jaeschke, S. Khan, J. R. Schneider, and J. B. Hastings (Springer International, New York, 2015), pp. 1-68 [arXiv:1504.01098v2].

[34] O. Zachar, S. A. Kivelson, and V. J. Emery, Landau Theory of Stripe Phases in Cuprates and Nickelates, Phys. Rev. B 57, 1422 (1998).

[35] See Supplemental Material at http://link.aps.org/ supplemental/10.1103/PhysRevX.8.011008 for details of the DFPT calculations and additional experimental data.

[36] B. Fåk and B. Dorner, Phonon Line Shapes and Excitation Energies, Physica B (Amsterdam) 234-236, 1107 (1997).

[37] See Sec. II of Ref. [35] for a discussion of the possible role of phonon splitting.

[38] X. M. Chen, V. Thampy, C. Mazzoli, A. M. Barbour, H. Miao, G. D. Gu, Y. Cao, J. M. Tranquada, M. P. M. Dean, and S. B. Wilkins, Remarkable Stability of Charge Density Wave Order in $\mathrm{La}_{1.875} \mathrm{Ba}_{0.125} \mathrm{CuO}_{4}$, Phys. Rev. Lett. 117, 167001 (2016).

[39] E. M. Forgan, E. Blackburn, A. T. Holmes, A. K. R. Briffa, J. Chang, L. Bouchenoire, S. D. Brown, R. Liang, D. Bonn, W. N. Hardy, N. B. Christensen, M. V. Zimmermann, M. Hücker, and S. M. Hayden, The Microscopic Structure of Charge Density Waves in Underdoped $\mathrm{YBa}_{2} \mathrm{Cu}_{3} \mathrm{O}_{6.54}$ Revealed by X-Ray Diffraction, Nat. Commun. 6, 10064 (2015).

[40] E. S. Božin, A. Huq, B. Shen, H. Claus, W. K. Kwok, and J. M. Tranquada, Charge-Screening Role of c-Axis Atomic Displacements in $\mathrm{YBa}_{2} \mathrm{Cu}_{3} \mathrm{O}_{6+x}$ and Related Superconductors, Phys. Rev. B 93, 054523 (2016).

[41] M. Hücker, M. V. Zimmermann, G. D. Gu, Z. J. Xu, J. S. Wen, G. Xu, H. J. Kang, A. Zheludev, and J. M. Tranquada, Stripe Order in Superconducting $\mathrm{La}_{2-x} \mathrm{Ba}_{x} \mathrm{CuO}_{4}$ (0.095 $\leq x \leq 0.155)$, Phys. Rev. B 83, 104506 (2011).

[42] S. B. Wilkins, M. P. M. Dean, J. Fink, M. Hücker, J. Geck, V. Soltwisch, E. Schierle, E. Weschke, G. Gu, S. Uchida, N. Ichikawa, J. M. Tranquada, and J. P. Hill, Comparison of Stripe Modulations in $\mathrm{La}_{1.875} \mathrm{Ba}_{0.125} \mathrm{CuO}_{4}$ and $\mathrm{La}_{1.48} \mathrm{Nd}_{0.4} \mathrm{Sr}_{0.12} \mathrm{CuO}_{4}$, Phys. Rev. B 84, 195101 (2011).

[43] M. P. M. Dean, G. Dellea, M. Minola, S. B. Wilkins, R. M. Konik, G. D. Gu, M. Le Tacon, N. B. Brookes, F. Yakhou-Harris, K. Kummer, J. P. Hill, L. Braicovich, and G. Ghiringhelli, Magnetic Excitations in Stripe-Ordered $\mathrm{La}_{1.875} \mathrm{Ba}_{0.125} \mathrm{CuO}_{4}$ Studied Using Resonant Inelastic X-Ray Scattering, Phys. Rev. B 88, 020403 (2013).

[44] V. Thampy, X. M. Chen, Y. Cao, C. Mazzoli, A. M. Barbour, W. Hu, H. Miao, G. Fabbris, R. D. Zhong, G. D. Gu, J. M. Tranquada, I. K. Robinson, S. B. Wilkins, and M. P. M. Dean, Static Charge Density Wave Order in the Superconducting State of $\mathrm{La}_{2-x} \mathrm{Ba}_{x} \mathrm{CuO}_{4}$, Phys. Rev. B 95, 241111(R) (2017).

[45] M. Hoesch, A. Bosak, D. Chernyshov, H. Berger, and M. Krisch, Giant Kohn Anomaly and the Phase Transition in Charge Density Wave $\mathrm{ZrTe}_{3}$, Phys. Rev. Lett. 102, 086402 (2009).

[46] U. Chatterjee, J. Zhao, M. Iavarone, R. Di Capua, J. P. Castellan, G. Karapetrov, C. D. Malliakas, M. G. Kanatzidis,
H. Claus, J.P.C. Ruff, F. Weber, J. van Wezel, J.C. Campuzano, R. Osborn, M. Randeria, N. Trivedi, M. R. Norman, and S. Rosenkranz, Emergence of Coherence in the Charge-Density Wave State of $2 \mathrm{H}-\mathrm{NbSe}_{2}$, Nat. Commun. 6, 6313 (2015).

[47] F. Weber, S. Rosenkranz, J.-P. Castellan, R. Osborn, R. Hott, R. Heid, K.-P. Bohnen, T. Egami, A. H. Said, and D. Reznik, Extended Phonon Collapse and the Origin of the ChargeDensity Wave in $2 \mathrm{H}-\mathrm{NbSe}_{2}$, Phys. Rev. Lett. 107, 107403 (2011).

[48] X. Zhu, Y. Cao, J. Zhang, E. W. Plummer, and J. Guo, Classification of Charge Density Waves Based on Their Nature, Proc. Natl. Acad. Sci. U.S.A. 112, 2367 (2015).

[49] H. Alloul, J. Bobroff, M. Gabay, and P. J. Hirschfeld, Defects in Correlated Metals and Superconductors, Rev. Mod. Phys. 81, 45 (2009).

[50] D. Haskel, E. A. Stern, F. Dogan, and A. R. Moodenbaugh, XAFS Study of the Low-Temperature Tetragonal Phase of $\mathrm{La}_{1.875} \mathrm{Ba}_{0.125} \mathrm{CuO}_{4}$ : Disorder, Stripes, and $T_{c}$ Suppression at $x=0.125$, Phys. Rev. B 61, 7055 (2000).

[51] L. Nie, A. V. Maharaj, E. Fradkin, and S. A Kivelson, Vestigial Nematicity from Spin and/or Charge Order in the Cuprates, Phys. Rev. B 96, 085142 (2017).

[52] Y. Wang and A. Chubukov, Charge-Density-Wave Order with Momentum $(2 q, 0)$ and $(0,2 q)$ within the SpinFermion Model: Continuous and Discrete Symmetry Breaking, Preemptive Composite Order, and Relation to Pseudogap in Hole-Doped Cuprates, Phys. Rev. B 90, 035149 (2014).

[53] Y.-H. Liu, R. M. Konik, T. M. Rice, and F. C. Zhang, Giant Phonon Anomaly Associated with Superconducting Fluctuations in the Pseudogap Phase of Cuprates, Nat. Commun. 7, 10378 (2016).

[54] E. H. da Silva Neto, B. Yu, M. Minola, R. Sutarto, E. Schierle, F. Boschini, M. Zonno, M. Bluschke, J. Higgins, Y. Li, G. Yu, E. Weschke, F. He, M. Le Tacon, R. L. Greene, M. Greven, G. A. Sawatzky, B. Keimer, and A. Damascelli, Doping-Dependent Charge Order Correlations in ElectronDoped Cuprates, Sci. Adv. 2, e1600782 (2016).

[55] T. Valla, A. V. Fedorov, J. Lee, J. C. Davis, and G. D. $\mathrm{Gu}$, The Ground State of the Pseudogap in Cuprate Superconductors, Science 314, 1914 (2006).

[56] J. Zaanen and P. B. Littlewood, Freezing Electronic Correlations by Polaronic Instabilities in Doped $\mathrm{La}_{2} \mathrm{NiO}_{4}$, Phys. Rev. B 50, 7222 (1994).

[57] M. Vojta, Lattice Symmetry Breaking in Cuprate Superconductors: Stripes, Nematics, and Superconductivity, Adv. Phys. 58, 699 (2009).

[58] L. K. Wagner, Ground State of Doped Cuprates from FirstPrinciples Quantum Monte Carlo Calculations, Phys. Rev. B 92, 161116 (2015).

[59] E. W. Huang, C. B. Mendl, S. Shenxiu Liu, S. Johnston, H. C. Jiang, B. Moritz, and T. P. Devereaux, Numerical Evidence of Fluctuating Stripes in the Normal State of High- $T_{c}$ Cuprate Superconductors, Science 358, 1161 (2017).

[60] A. Mesaros, K. Fujita, S. D. Edkins, M. H. Hamidian, H. Eisaki, S.-i. Uchida, J. C. Samus Davis, M. J. Lawler, and E.-A. Kim, Commensurate $4 a_{0}$-Period Charge Density Modulations throughout the $\mathrm{Bi}_{2} \mathrm{Sr}_{2} \mathrm{CaCu}_{2} \mathrm{O}_{8+x}$ Pseudogap Regime, Proc. Natl. Acad. Sci. U.S.A. 113, 12661 (2016). 
[61] M. Kato, K. Machida, H. Nakanishi, and M. Fujita, Soliton Lattice Modulation of Incommensurate Spin Density Wave in Two Dimensional Hubbard Model-A Mean Field Study, J. Phys. Soc. Jpn. 59, 1047 (1990).

[62] M. Hücker, M. v. Zimmermann, Z. J. Xu, J. S. Wen, G. D. $\mathrm{Gu}$, and J. M. Tranquada, Enhanced Charge Stripe Order of Superconducting $\mathrm{La}_{2-x} \mathrm{Ba}_{x} \mathrm{CuO}_{4}$ in a Magnetic Field, Phys. Rev. B 87, 014501 (2013).

[63] O. Zachar, Stripes Disorder and Correlation Lengths in Doped Antiferromagnets, Phys. Rev. B 62, 13836 (2000).
[64] H. Kimura, H. Matsushita, K. Hirota, Y. Endoh, K. Yamada, G. Shirane, Y. S. Lee, M. A. Kastner, and R. J. Birgeneau, Incommensurate Geometry of the Elastic Magnetic Peaks in Superconducting $\mathrm{La}_{1.88} \mathrm{Sr}_{0.12} \mathrm{CuO}_{4}$, Phys. Rev. B 61, 14366 (2000).

[65] V. Thampy, S. Blanco-Canosa, M. Garcia-Fernandez, M. P. M. Dean, G. D. Gu, M. Först, T. Loew, B. Keimer, M. Le Tacon, S. B. Wilkins, and J. P. Hill, Comparison of Charge Modulations in $\mathrm{La}_{1.875} \mathrm{Ba}_{0.125} \mathrm{CuO}_{4}$ and $\mathrm{YBa}_{2} \mathrm{Cu}_{3} \mathrm{O}_{6.6}$, Phys. Rev. B 88, 024505 (2013). 